\title{
Some exact results in chiral gauge theories
}

\author{
Csaba Csáki, ${ }^{1, *}$ Hitoshi Murayama ${ }^{2,3,4, \dagger}$ and Ofri Telem $\oplus^{2,4, \$}$ \\ ${ }^{1}$ Department of Physics, LEPP, Cornell University, Ithaca, New York 14853, USA \\ ${ }^{2}$ Department of Physics, University of California, Berkeley, California 94720, USA \\ ${ }^{3}$ Kavli Institute for the Physics and Mathematics of the Universe (WPI), University of Tokyo, \\ Kashiwa 277-8583, Japan \\ ${ }^{4}$ Ernest Orlando Lawrence Berkeley National Laboratory, Berkeley, California 94720, USA
}

(Received 7 May 2021; accepted 30 August 2021; published 27 September 2021)

\begin{abstract}
We analyze dynamics of chiral gauge theories based on the $S U(N)$ gauge group with one antisymmetric tensor $A$ and $(N-4)$ antifundamentals $F_{i}$ when $N$ is odd. Based on the continuity to the supersymmetric gauge theories with anomaly-mediated supersymmetry breaking, we claim that the global $S U(N-4)$ symmetry is spontaneously broken to $S p(N-5)$. There are $N-5$ massless fermions as a fundamental representation of $S p(N-5)$, and another massless fermion, together saturating the anomaly matching conditions. When $N$ is even, the unbroken flavor symmetry is $\operatorname{Sp}(N-4)$ while there are no massless fermions. Our result is different from the dynamics suggested by tumbling where the full $S U(N-4)$ symmetry is unbroken, but the tumbling picture can be modified via the addition of a second condensate to produce the symmetry breaking pattern predicted from our method.
\end{abstract}

DOI: 10.1103/PhysRevD.104.065018

\section{INTRODUCTION}

Non-Abelian gauge theories [1] are the basis of our modern understanding of microscopic physics. Quantum chromodynamics (QCD) based on the $S U(3)$ gauge group is a prime example with vectorlike particle content. In general, $S U(N)$ gauge theories with $N_{f}$ quarks in the fundamental representation are called QCD-like theories. Inspired by the light pions and Bardeen-Cooper-Schrieffer (BCS) theory of superconductivity [2], Nambu and JonaLasinio conjectured dynamical chiral symmetry breaking $[3,4]$, which is now believed to be the correct dynamics of QCD-like theories.

However, the dynamics of chiral gauge theories are difficult to understand. There has been theoretical progress in representing chiral gauge theories on the lattice [5-13], which is a potential avenue for future numerical simulations; yet they are numerically expensive and progress is slow. Arguably, understanding the dynamics of chiral gauge theories is one of the most important open questions in quantum field theories.

\footnotetext{
*ccsaki@gmail.com

hitoshi@berkeley.edu, hitoshi.murayama@ipmu.jp

t10ofrit@gmail.com
}

Published by the American Physical Society under the terms of the Creative Commons Attribution 4.0 International license. Further distribution of this work must maintain attribution to the author(s) and the published article's title, journal citation, and DOI. Funded by SCOAP ${ }^{3}$.
While there is no established systematic approach there does exist a conjectured framework for the dynamics of chiral gauge theories called tumbling [14]. It postulates certain fermion bilinear condensates that dynamically break the gauge symmetry until the remaining gauge group becomes QCD-like. For example, an $S U(N)$ gauge theory with an antisymmetric tensor $A$ and $(N-4)$ antifundamentals $\bar{F}_{i}$ was argued [15] to break the gauge symmetry by the condensate $\left\langle A^{a b} \bar{F}_{i}^{b}\right\rangle=v^{3} \delta_{i}^{a} \neq 0$ to an $S U(4)$ gauge theory that confines. It assumes massless symmetric tensor composite fermions $A \bar{F}_{\{i,} \bar{F}_{j\}}$. Even though the conjecture satisfies nontrivial 't Hooft anomaly matching conditions, it has never been clear if it is the correct understanding. Recently, these proposals have undergone further scrutiny in $[16,17]$, by applying new discrete anomaly matching conditions [18] involving the center symmetry $Z_{n}$ of the gauge group, in the spirit of [19-21]. We do not elaborate more on these generalized consistency conditions, since they seem to automatically hold for our proposal of the IR dynamics, due to its continuous connection to the supersymmetric theory.

In [22], a novel approach was proposed to study the dynamics of nonsupersymmetric gauge theories via anomalymediated supersymmetry breaking (AMSB) [23,24]. It is based on the Weyl compensator field

$$
\Phi=1+\theta^{2} m,
$$

where $m$ dictates the size of the supersymmetry (SUSY) breaking. The UV theory has mass for squarks and 
gauginos, which decouple from dynamics when $m$ is increased, and it is therefore continuously connected to nonsupersymmetric gauge theories. Due to the ultraviolet insensitivity of the AMSB [25,26], the dynamics can be studied using the particle content and interactions at each energy scale. In particular, a consistent picture was obtained for QCD-like theories [22]. For other approaches to extrapolating from supersymmetric theories to their nonSUSY counterparts, see, for example, [27,28].

In this paper, we apply the AMSB methodology to analyze the dynamics of nonsupersymmetric chiral gauge theories. We begin the discussion with the simplest and most well-known chiral gauge theory; $S U(5)$ with an antisymmetric tensor and an antifundamental Weyl fermion. Its supersymmetric version is well known to break SUSY dynamically, though the actual dynamics is not calculable. We point out that in the SUSY breaking minimum we expect a massless composite fermion, which is expected to persist in the nonsupersymmetric theory after adding AMSB. Next we analyze the general $S U(N)$ $(N=2 n+1$ odd) theories with an antisymmetric tensor and $N-4$ antifundamentals. Again the SUSY version with AMSB can be worked out exactly, leading to the dynamical breaking of the $S U(N-4) \times U(1)$ global symmetry to $S p(N-5) \times U(1)$, as well as massless fermions in the fundamental and singlet representations of $S p(N-5)$. This picture continuously connects to the non-SUSY limit, while it does not agree with the simplest tumbling predictions. We show however that one can extend the tumbling picture by adding another condensate in the second most attractive channel to obtain a symmetry breaking pattern that agrees with the non-SUSY limit of the AMSB approach. Finally we also discuss the case of even $N=2 n$ with no massless fermions content.

\section{ANOMALY MEDIATION}

Anomaly mediation of supersymmetry breaking is parametrized by a single number $m$ that explicitly breaks supersymmetry in two different ways. One is the tree-level contribution based on the superpotential

$$
\mathcal{L}_{\text {tree }}=m\left(\phi_{i} \frac{\partial W}{\partial \phi_{i}}-3 W\right)+\text { c.c. }
$$

The other is the loop-level supersymmetry breaking effects in trilinear couplings, scalar masses, and gaugino masses,

$$
\begin{gathered}
A_{i j k}(\mu)=-\frac{1}{2}\left(\gamma_{i}+\gamma_{j}+\gamma_{k}\right)(\mu) m, \\
m_{i}^{2}(\mu)=-\frac{1}{4} \dot{\gamma}_{i}(\mu) m^{2}, \\
m_{\lambda}(\mu)=-\frac{\beta\left(g^{2}\right)}{2 g^{2}}(\mu) m .
\end{gathered}
$$

Here, $\gamma_{i}=\mu \frac{d}{d \mu} \ln Z_{i}(\mu), \dot{\gamma}=\mu \frac{d}{d \mu} \gamma_{i}$, and $\beta\left(g^{2}\right)=\mu \frac{d}{d \mu} g^{2}$. When the gauge theory is asymptotically free, $m_{i}^{2}>0$ which stabilizes the theory against runaway behaviors. Note that Eq. (1) also breaks the $U(1)_{R}$ symmetry explicitly and hence we do not need to study its anomaly matching conditions.

\section{III. $S U(5)$ WITH $A(10)$ AND $\bar{F}(\overline{5})$}

This is the simplest and most well-known chiral SUSY gauge theory which has a nonanomalous $U(1)_{5}$ symmetry $A(+1), \bar{F}(-3)$ (see Table I). This theory breaks supersymmetry dynamically [29]. (See [30] for how this theory is related to the 4-1 and 3-2 models.) Even though the theory is intrinsically strongly coupled, it was shown that adding a pair of $F+\bar{F}$ allows for a weakly-coupled analysis [31] (see also [32,33]). Once a mass term is added to the pair, one can explicitly calculate the actual SUSY breaking minimum of the potential. (See the Supplemental material [34] for details of the analysis.)

Surprisingly, one of the most important features of the SUSY breaking minimum has so far (to the best of our knowledge) not been discussed in the literature. We are finding that in spite of the strong dynamics the $U(1)_{5}$ global symmetry remains unbroken, implying the presence of a massless fermion that can be identified with the composite fermion $A \bar{F} \bar{F}$ [35]. This massless fermion exists in addition to the massless Goldstino required for SUSY breaking. It has charge -5 and one can readily check that it saturates all 't Hooft anomalies

$$
\begin{aligned}
U(1)_{5} \text { gravity }^{2}: & \mathbf{1 0}(+1)+\overline{\mathbf{5}}(-3)=(-5), \\
U(1)_{5}^{3}: & \mathbf{1 0}(+1)^{3}+\overline{\mathbf{5}}(-3)^{3}=(-5)^{3} .
\end{aligned}
$$

This nontrivial 't Hooft anomaly matching is already a very strong argument in favor of the existence of the unbroken $U(1)_{5}$ symmetry, which as explained above can be independently verified by analyzing the theory with the extra flavor added, which is done explicitly in the Supplemental material [34].

Since supersymmetry is already spontaneously broken, adding small explicit supersymmetry breaking via AMSB does not change the dynamics, while the goldstino acquires a mass. On the other hand, the other massless fermion $A \bar{F} \bar{F}$ is protected because of the anomaly matching condition

TABLE I. Particle content of the $S U(5)$ theory with one antisymmetric $A$ and one antifundamental $\bar{F}$. The composite fermion $A \bar{F} \bar{F}$ matches all anomalies.

\begin{tabular}{lcc}
\hline \hline & $S U(5)$ & $U(1)_{5}$ \\
\hline$A$ & $\square$ & +1 \\
$\bar{F}$ & $\square$ & -3 \\
$A \bar{F} \bar{F}$ & 1 & -5 \\
\hline \hline
\end{tabular}


and remains massless even in the nonsupersymmetric theory. The appearance of the unbroken $U(1)_{5}$ and the corresponding massless fermion is our first example of exact results in chiral gauge theories. Extrapolating $m \gg \Lambda$, we expect the same massless fermion remaining in the theory while the goldstino decouples.

Interestingly, in this particular case our analysis is consistent with results suggested by tumbling $[14,36]$. In that approach one would postulate that the operator $\epsilon_{a b c d e} A^{b c} A^{d e}$ corresponding to the most attractive channel (MAC) condensate [37], breaking the $S U(5)$ gauge symmetry to $S U(4)$, while retaining a global $U(1)$. Under the unbroken $S U(4)_{\text {gauge }} \times U(1)_{\text {global }}, A$ decomposes as $\mathbf{6}_{0}+$ $\mathbf{4}_{-5 / 2}$ while $\bar{F}$ as $\overline{\mathbf{4}}_{5 / 2}+\mathbf{1}_{-5}$. The degrees of freedom charged under $S U(4)$ are vectorlike, and so they condense just like in QCD and become massive. However, $\mathbf{1}_{-\mathbf{5}}$ is chiral, and remains as a massless fermion, which can be identified with the composite $A\left(\mathbf{4}_{-5 / 2}\right) \bar{F}\left(\mathbf{4}_{5 / 2}\right) \bar{F}\left(\mathbf{1}_{-5}\right)$. Note that for general $S U(N)$ our method leads to predictions that differ somewhat from those in [14]. However we will see that the tumbling scheme can be augmented to produce results consistent with ours.

\section{IV. $S U(N)$ WITH $A$ AND $(N-4) \bar{F}$}

Next we consider the generalization of the above $S U(5)$ theory to $S U(N)$ with $N=2 n+1$ odd. Table II shows the matter content and the symmetries of the theory. In the SUSY limit, the theory has the $D$-flat direction

$$
\begin{gathered}
A A^{\dagger}+A^{\dagger} A-\bar{F}_{i}^{*} \bar{F}_{i}^{T}=0, \\
A=\frac{\varphi}{\sqrt{2}}\left(\begin{array}{c|c}
J_{(N-5)} & 0 \\
\hline 0 & 0_{5 \times 5}
\end{array}\right), \quad \bar{F}=\varphi\left(\begin{array}{c|c}
I_{(N-5)} & 0 \\
\hline 0 & 0_{5 \times 1}
\end{array}\right),
\end{gathered}
$$

where $J_{(N-5)}=i \sigma_{2} \otimes I_{(N-5) / 2}$. As we will see below, in our scenario the flat direction will ultimately be stabilized at large vacuum expectation values (VEVs), and so a

TABLE II. Particle content of the $S U(N)$ theory with one antisymmetric $A$ and $N-4$ antifundamentals $\bar{F}_{i} . N=2 n+1$ is odd. The operators $A \bar{F}_{i} \bar{F}_{N-4}$ are interpolating fields for massless fermions. Decompositions of fields under the unbroken $\operatorname{Sp}(N-$ $5) \times U(1)^{\prime}$ symmetry are also shown. The $U(1)^{\prime}$ charges are given separately for the fundamental and singlet of the unbroken $S p(N-5)$ global symmetry.

\begin{tabular}{lccccc}
\hline \hline & $S U(N)$ & $U(1)$ & $S U(N-4)$ & $S p(N-5)$ & $U(1)^{\prime}$ \\
\hline$A$ & $\square$ & $-N+4$ & 1 & 1 & $-N+4$ \\
& & & & $\square$ & $\frac{1}{2}(N-4)$ \\
$\bar{F}_{i}$ & $\square$ & $N-2$ & $\square$ & 1 & $\frac{(N+1)(N-4)}{2}$ \\
& & & & $\square$ & $\frac{N(N-4)}{2}$ \\
$A \bar{F}_{i} \bar{F}_{N-4}$ & $\mathbf{1}$ & $N$ & $\square$ & 1 & $N(N-4)$ \\
\hline \hline
\end{tabular}

weakly coupled analysis is appropriate here. Along the flat directions the gauge symmetry is broken to $S U(5)$ while the $S U(N-4)$ global symmetry is broken to $S p(N-5)$. This is easily seen from the fact that

$$
A \bar{F}_{i} \bar{F}_{j}=\frac{1}{\sqrt{2}} \varphi^{3} J_{i j}, \quad i, j \leq N-5,
$$

on the flat directions. There is also an unbroken $U(1)^{\prime}$ global symmetry whose generator is $Q^{\prime}=Q-\frac{N}{2} T_{N-4}$ where $Q$ is the generator of the original $U(1)$. Here $T_{N-4}=\operatorname{diag}(1 \ldots, 1,-(N-5), 0,0,0,0,0)$, is an unnormalized generator in $S U(N-4) / S p(N-5)$. The dynamical scale of the unbroken $S U(5)$ is given by

$$
\Lambda_{5}^{13}=\frac{\Lambda_{N}^{2 N+3}}{\left(\operatorname{Pf}^{\prime} A \bar{F} \bar{F}\right)\left(\operatorname{Pf}^{\prime} A\right)},
$$

where the Pfaffians involve only the $N-5$ components, as indicated by the prime. This unbroken $S U(5)$ will have the same matter content as in the previous example, hence it confines and breaks supersymmetry at the scale $\Lambda_{5}$. Here and below, we absorb renormalization-scheme-dependent numerical constants [39] into the definition of the scale $\Lambda$ 's which does not affect any of the discussions below. The vacuum energy has a runaway dependence on $\varphi$

$$
V \approx \Lambda_{5}^{4}=\left(\frac{\Lambda_{N}^{2 N+3}}{\varphi^{2 N-10}}\right)^{4 / 13},
$$

without a stable ground state. It can be stabilized by adding $\lambda A \bar{F}_{i} \bar{F}_{j} J^{i j}$ to the superpotential [29].

With AMSB there is no need to add a tree-level superpotential, since the run-away behavior is stabilized by the scalar masses-squared along the flat direction

$$
m_{A, \bar{F}_{i}}^{2}=\frac{g^{4}}{\left(8 \pi^{2}\right)^{2}} 2 C_{i}(2 N+3) m^{2},
$$

where

$$
C_{i}= \begin{cases}\frac{(N+1)(N-2)}{N} & \text { for } A, \\ \frac{N^{2}-1}{2 N} & \text { for } \bar{F}_{i} .\end{cases}
$$

Note that Eq. (11) is a runaway potential from dynamical supersymmetry breaking, not a superpotential. Therefore, there is no accompanying tree-level AMSB piece from (2) to stabilize it. This is a key difference from the Affleck-Dine-Seiberg case of QCD-like theories [22], and from the even- $N$ case discussed in the next section. The theory has a stable ground state at

$$
\varphi \approx \Lambda\left(\frac{4 \pi \Lambda}{m}\right)^{13 /(4 N-7)} \gg \Lambda .
$$


Therefore, the physics is weakly coupled at the scale $\varphi$ and our analysis is justified.

We now present a heuristic description of the dynamics in the Higgs picture, before passing to a purely gauge invariant formulation below. In this picture the UV theory has $A\left(\frac{1}{2} \mathbf{N}(\mathbf{N}-\mathbf{1})\right)+\bar{F}(\overline{\mathbf{N}}) \times(N-4)$ chiral superfields. $N^{2}-1-24$ are eaten when the $S U(N)$ gauge symmetry is Higgsed to $S U(5)$. Of the remaining ones, $15=A(\mathbf{1 0})+$ $\bar{F}_{N-4}(\overline{\mathbf{5}})$ are charged under the gauge $S U(5)$, and the other $\frac{1}{2}(N-4)(N-5)$ contain the Nambu-Goldstone bosons for the broken global symmetry [40].

The $S U(5)$ dynamics with $A(\mathbf{1 0})$ and $\bar{F}_{N-4}(\overline{\mathbf{5}})$ is along the lines of the previous section; $S U(5)$ is Higgsed to $S U(4)$, supersymmetry is spontaneously broken, and there is a massless goldstino and an additional massless fermion. Anomaly matching is then satisfied by this fermion, together with the fermions in the first $N-5$ components of $\bar{F}_{N-4}$, which are neutral under the unbroken $S U(5)$ gauge group and remain massless.

In gauge invariant language, the IR theory has $N-4$ massless fermions $A \bar{F}_{i} \bar{F}_{N-4}$ [42] in the singlet and fundamental of the unbroken $S p(N-5)$ global symmetry. These correspond to the $N-5+1$ massless fermions of the Higgs picture.

The massless fermions match all of the anomalies for the unbroken $S p(N-5) \times U(1)^{\prime} ; U(1)^{\prime}$ gravity $^{2}$ :

$$
\begin{aligned}
& \frac{\mathbf{1}}{\mathbf{2}} \mathbf{N}(\mathbf{N}-\mathbf{1})(-N+4)+\overline{\mathbf{N}}(N-5) \frac{1}{2}(N-4) \\
& \quad+\overline{\mathbf{N}} \frac{1}{2}(N+1)(N-4) \\
& =(N-5) \frac{1}{2} N(N-4)+N(N-4),
\end{aligned}
$$

$U(1)^{13}$ :

$$
\begin{aligned}
& \frac{\mathbf{1}}{\mathbf{2}} \mathbf{N}(\mathbf{N}-\mathbf{1})(-N+4)^{3}+\overline{\mathbf{N}}(N-5)\left(\frac{1}{2}(N-4)\right)^{3} \\
& \quad+\overline{\mathbf{N}}\left(\frac{1}{2}(N+1)(N-4)\right)^{3} \\
& =(N-5)\left(\frac{1}{2} N(N-4)\right)^{3}+(N(N-4))^{3},
\end{aligned}
$$

$U(1)^{\prime} S p(N-5)^{2}$ :

$$
\overline{\mathbf{N}} \frac{1}{2}(N-4)=\frac{1}{2} N(N-4),
$$

and $S p(N-5)_{\text {Witten }}$ :

$$
\overline{\mathbf{N}}=1(\bmod 2) .
$$

In addition, there are massless Nambu-Goldstone bosons of the $[S U(N-4) \times U(1)] /\left[S p(N-5) \times U^{\prime}(1)\right]$ coset space, together with the Wess-Zumino-Witten term $[43,44]$ given that $\pi_{5}(S U(N-4) / S p(N-5))=\mathbb{Z}$ for the $S U(N-4)$ anomalies not contained in $S p(N-5)$ induced by the one-loop diagrams of massive fermions [45]. This is the second example of exact results on chiral gauge theories.

When $m$ is increased, the field values approach the strong scale, and we lose control of the dynamics. The nontrivial anomaly matching conditions depend only on the presence of the massless fermions and may well persist to the limit $m \gg \Lambda$. While there may be a phase transition that lifts the massless fermions discontinuously, our analysis provides a concrete suggestion for the dynamics of the nonsupersymmetric chiral gauge theory, which should be checked explicitly by lattice methods. It would also be interesting to see whether the entire chiral compensator $\Phi$ could be embedded in a fully supersymmetric theory with spontaneous SUSY breaking, giving rise to the scale $m$ governed by some holomorphic parameters of the UV complete theory. In that case one may perhaps argue against the presence of a phase transition in the $m \rightarrow \infty$ limit.

\section{COMPARISON TO TUMBLING}

While there is no controlled analysis for the study of the dynamics of chiral nonsupersymmetric gauge theories, there is a framework proposed by Raby, Dimopoulos, and Susskind [14] that goes broadly under the name of "tumbling". One first finds the MAC among the charged fields and assumes that it condenses, breaking part of the gauge symmetry. This process is then iterated until one arrives at a QCD-like theory (or when the gauge group is fully broken). Applying this method of tumbling to the nonsupersymmetric $S U(N)$ theory with chiral fermions $A(\square)+(N-4) \bar{F}(\bar{\square})$, one finds [15] that the most attractive channel is the antisymmetric tensor $A^{a b}$ and the antifundamentals $\bar{F}_{b i}$ combined into fundamentals. This leads to a condensate

$$
\left\langle A^{a b} \bar{F}_{b i}\right\rangle \sim \Lambda^{3} \delta_{i}^{a} \neq 0, \quad i, a \leq N-4 .
$$

The above condensate breaks the $S U(N)$ gauge symmetry down to $S U(4)$ while locking $N-4$ colors and flavors. This leaving unbroken a global $S U(N-4)$, which is the diagonal combination of the $S U(N)$ gauge group and the $S U(N-4)$ global symmetry. The remaining $S U(4)$ symmetry is vectorlike and hence assumed confining. The global anomalies of the unbroken $S U(N-4)$ symmetry are matched by a massless fermion in the symmetric tensor representation of $S U(N-4)$ corresponding to the fermionic composite $A \bar{F}_{\{i,} \bar{F}_{j\}}$. While this picture appears possible, there are no controlled limits where the theory can be studied reliably along this line of analysis.

Comparing to the results of our nonsupersymmetric AMSB limit we can see that the above tumbling picture indeed appears to be incomplete. For the SUSY + AMSB 
case we have found that the remaining global symmetry is $S p(N-5) \times U(1)^{\prime}$ instead of the full $S U(N-4) \times U(1)$, with two massless fermion composites. It is actually easy to reconcile the tumbling picture with our SUSY-inspired predictions. One needs to simply consider the second most attractive channel corresponding to two antifundamentals in the antisymmetric combination, and assume another condensate along this direction,

$$
\left\langle\bar{F}_{a i} \bar{F}_{b j}\right\rangle \sim \Lambda^{3} J_{a b} J_{i j} \neq 0, \quad 1 \leq i, j, a, b \leq N-5,
$$

in addition to the one in Eq. (19). Since the gauge indices are antisymmetric between $a$ and $b$ to make this channel attractive, the flavor indices $i$ and $j$ will also have to be antisymmetric. Note that the symmetric combination $\delta_{a b} \delta_{i j}$ is repulsive and no condensate along that direction is expected. The condensate Eq. (20) breaks the global $S U(N-4)$ symmetry left by Eq. (19) further down to $\operatorname{Sp}(N-5)$.

The condensate in Eq. (20) is a good description in the weakly-coupled Higgs picture. When we increase $m$, the theory becomes strongly coupled and this description is no longer valid. Instead, we should refer to a gauge invariant order parameter for a consistent description, which in our case is

$$
\left\langle\left(\bar{F}_{i} \bar{F}_{j}\right)_{[a, b]}\left(\bar{F}^{* k} \bar{F}^{* l}\right)^{[a, b]}\right\rangle \propto J_{i j} J^{k l}, 1 \leq i, j, k, l \leq N-5 .
$$

The candidate Nambu-Goldstone bosons eaten by the $S U(N) / S U(4)$ massive gauge bosons are $A^{a b} \bar{F}_{b i}$ for the upper $(N-4)$-dimensional block and $A^{p b} \bar{F}_{b i}$ for the offdiagonal block where $p=1, \ldots, 4$ denotes the $S U(4)$ index. Finally, $J^{a b} \bar{F}_{a i} \bar{F}_{b j}$ are the candidates for the uneaten Nambu-Goldstone bosons for the global $S U(N-4)$ / $S p(N-5)$ coset.

The condensate $A^{a b} \bar{F}_{b i}$ separates the $\bar{F}_{i}$ into the first $\bar{F}_{1 \ldots(N-5)}$ components and $\bar{F}_{N-4}$. These end up in the $S p(N-5)$ fundamental and singlet parts of the IR composite fermions $A \bar{F}_{i} \bar{F}_{N-4}$ (see Table II for their charges). Under the unbroken $S U(4)$ symmetry, the charged fermions decompose as $A(\mathbf{6}+\mathbf{4})$ and $\bar{F}_{i}(\overline{\mathbf{4}})$. These remaining degrees of freedom are vectorlike, and become massive. The anomaly matching conditions remain the same and satisfied exactly as in the case of SUSY + AMSB. Therefore, this modified tumbling picture with the second condensate has the identical symmetry breaking pattern and massless fermion content as the SUSY theory with the AMSB, hence we find the two to be likely continuously connected. We expect this modified picture to provide the proper low-energy dynamics of the nonsupersymmetric theory.

\section{EVEN $N$}

Once again, we consider $S U(N)$ gauge theories with an antisymmetric tensor $A$ and $(N-4)$ antifundamentals $\bar{F}_{i}$,
TABLE III. Particle content of the $S U(N)$ theory with one antisymmetric $A$ and $N-4$ antifundamentals $\bar{F}_{i} . N=2 n$ is even. Decompositions under the unbroken $S p(N-4)$ symmetry are also shown.

\begin{tabular}{lcccc}
\hline \hline & $S U(N)$ & $U(1)$ & $S U(N-4)$ & $S p(N-4)$ \\
\hline$A$ & $\boxminus$ & $-N+4$ & $\mathbf{1}$ & $\mathbf{1}$ \\
$\bar{F}_{i}$ & $\square$ & $N-2$ & $\square$ & $\square$ \\
$A \bar{F}_{i} \bar{F}_{j}$ & $\mathbf{1}$ & $N$ & $\square$ & $\boxminus \oplus \mathbf{1}$ \\
$\operatorname{Pf} A$ & $\mathbf{1}$ & $-\frac{1}{2} N(N-4)$ & $\mathbf{1}$ & $\mathbf{1}$ \\
\hline \hline
\end{tabular}

but for even $N=2 n$. (See Table III for quantum numbers.) In this case, the $D$-flat directions break the gauge group to $S p(4)=S O(5)$ (see [46] and also the Supplemental material [34]), whose gaugino condensate induces a dynamical superpotential $[32,33,46]$,

$$
W=\left(\frac{\Lambda^{2 N+3}}{(\operatorname{Pf} A \bar{F} \bar{F})(\operatorname{Pf} A)}\right)^{1 / 3} .
$$

The AMSB Eq. (2) balances the superpotential against the supersymmetry breaking such that both $A \sim \bar{F} \sim$ $\Lambda(\Lambda / m)^{3 / 2 N}$ and all fermions acquire mass. The global $S U(N-4)$ and $U(1)$ symmetries are broken dynamically to $S p(N-4)$ which does not have anomalies. The anomalies of broken symmetries are saturated by the NambuGoldstone bosons with the Wess-Zumino-Witten term given $\pi_{5}(S U(N-4) / S p(N-4))=\mathbb{Z}$. This is the third example of exact results on chiral gauge theories.

Again this dynamics can persist to $m \gg \Lambda$. We can interpret the dynamics in the non-SUSY limit with the fermion bilinear condensates

$$
\begin{aligned}
\left\langle A^{a b} \bar{F}_{b i}\right\rangle & \sim \Lambda^{3} \delta_{i}^{a}, \\
\left\langle\bar{F}_{a i} \bar{F}_{b j}\right\rangle & \sim \Lambda^{3} J_{a b} J_{i j}
\end{aligned} \quad i, j, a, b \leq N-4 .
$$

Note that in this case $\bar{F}_{N-4}$ is not singled out by the condensate, and there are no corresponding massless fermions. The remaining theory is $S p(4)$ with $A(\mathbf{6}+\mathbf{4})$ and $\bar{F}(\mathbf{4})$ which is vectorlike and becomes massive. The massless degrees of freedom are chiral Lagrangians of $S U(N-4) \times U(1) / S p(N-4)$ with the Wess-ZuminoWitten term.

\section{CONCLUSIONS}

In this paper, we outlined how dynamics of chiral gauge theories can be studied by perturbing the supersymmetric version with anomaly-mediated supersymmetry breaking. In particular, we worked out dynamics of $S U(N)$ gauge theories with an antisymmetric tensor and $N-4$ antifundamentals. We came up with a consistent picture that connects supersymmetric gauge theories perturbed by the anomaly-mediated supersymmetry breaking to 
nonsupersymmetric gauge theories. The symmetry breaking pattern suggested differs from that based on the original tumbling argument, which however can be extended to match the picture obtained here. It would be interesting to extend this analysis to other examples of chiral gauge theories. Ultimately, lattice gauge theory simulations will have the final verdict on the picture.

\section{ACKNOWLEDGMENTS}

We thank Yuri Shirman for careful reading of the manuscript and useful comments. C. C. is supported in part by the NSF Grant No. PHY-2014071 as well as the USIsraeli BSF Grant No. 2016153. We also thank Jacob Leedom for pointing out typos. O.T. and H. M. were supported in part by the DOE under Grant No. DE-AC0205CH11231. H. M. was also supported in part by the NSF Grant No. PHY-1915314, by the JSPS Grant-in-Aid for Scientific Research JP20K03942, MEXT Grant-in-Aid for Transformative Research Areas (A) JP20H05850, JP20A203, by the World Premier International Research Center Initiative, MEXT, Japan, and Hamamatsu Photonics, K. K.
[1] C.-N. Yang and R. L. Mills, Phys. Rev. 96, 191 (1954).

[2] J. Bardeen, L. N. Cooper, and J. R. Schrieffer, Phys. Rev. 108, 1175 (1957).

[3] Y. Nambu and G. Jona-Lasinio, Phys. Rev. 122, 345 (1961).

[4] Y. Nambu and G. Jona-Lasinio, Phys. Rev. 124, 246 (1961).

[5] P. H. Ginsparg and K. G. Wilson, Phys. Rev. D 25, 2649 (1982).

[6] D. B. Kaplan, Phys. Lett. B 288, 342 (1992).

[7] Y. Shamir, Nucl. Phys. B406, 90 (1993).

[8] R. Narayanan and H. Neuberger, Nucl. Phys. B443, 305 (1995).

[9] M. Luscher, Phys. Lett. B 428, 342 (1998).

[10] M. Golterman, Nucl. Phys. B, Proc. Suppl. 94, 189 (2001).

[11] M. Golterman and Y. Shamir, Phys. Rev. D 70, 094506 (2004).

[12] D. M. Grabowska and D. B. Kaplan, Phys. Rev. Lett. 116, 211602 (2016).

[13] D. M. Grabowska and D. B. Kaplan, Phys. Rev. D 94, 114504 (2016).

[14] S. Raby, S. Dimopoulos, and L. Susskind, Nucl. Phys. B169, 373 (1980).

[15] S. Dimopoulos, S. Raby, and L. Susskind, Nucl. Phys. B173, 208 (1980).

[16] S. Bolognesi, K. Konishi, and A. Luzio, J. High Energy Phys. 09 (2020) 001.

[17] S. Bolognesi, K. Konishi, and A. Luzio, Phys. Rev. D 103, 094016 (2021).

[18] C. Csáki and H. Murayama, Nucl. Phys. B515, 114 (1998).

[19] D. Gaiotto, A. Kapustin, Z. Komargodski, and N. Seiberg, J. High Energy Phys. 05 (2017) 091.

[20] Y. Tanizaki, J. High Energy Phys. 08 (2018) 171.

[21] S. Bolognesi, K. Konishi, and A. Luzio, J. High Energy Phys. 01 (2020) 048.

[22] H. Murayama, Phys. Rev. Lett. 126, 251601 (2021).

[23] L. Randall and R. Sundrum, Nucl. Phys. B557, 79 (1999).

[24] G. F. Giudice, M. A. Luty, H. Murayama, and R. Rattazzi, J. High Energy Phys. 12 (1998) 027.

[25] A. Pomarol and R. Rattazzi, J. High Energy Phys. 05 (1999) 013.

[26] E. Boyda, H. Murayama, and A. Pierce, Phys. Rev. D 65, 085028 (2002).
[27] O. Aharony, J. Sonnenschein, M. E. Peskin, and S. Yankielowicz, Phys. Rev. D 52, 6157 (1995).

[28] H.-C. Cheng and Y. Shadmi, Nucl. Phys. B531, 125 (1998).

[29] I. Affleck, M. Dine, and N. Seiberg, Phys. Lett. 137B, 187 (1984).

[30] R. G. Leigh, L. Randall, and R. Rattazzi, Nucl. Phys. B501, 375 (1997).

[31] H. Murayama, Phys. Lett. B 355, 187 (1995).

[32] E. Poppitz and S. P. Trivedi, Phys. Lett. B 365, 125 (1996).

[33] P. Pouliot, Phys. Lett. B 367, 151 (1996).

[34] See Supplemental Material at http://link.aps.org/ supplemental/10.1103/PhysRevD.104.065018 for derivation of the IR structure of supersymmetric $S U(5)$ with one matter field in the antisymmetric tensor and one antifundamental, in the presence of AMSB. We did this by adding and decoupling a flavor $F+\bar{F}$. We found an unbroken global $U(1)$ in the IR, whose "t Hooft anomalies are matched by a composite fermion $A \bar{F} \bar{F}$, in addition to a Goldstino from dynamical SUSY breaking which decouples when the AMSB is large. We also provided more details on the D-flat directions for $S U(N)$ with an antisymmetric and $N-4$ antifundamentals, when $N$ is even.

[35] More explicitly, the massless fermion is given by $\left(\epsilon^{\alpha \beta} A_{\alpha}^{a b} \bar{F}_{2 a \beta}\right) \bar{F}_{2 b \gamma}$, where $\alpha, \beta, \gamma=1,2$ are spinor indices.

[36] H. Georgi, Nucl. Phys. B156, 126 (1979).

[37] When discussing the tumbling approach, we will use the heuristic notion of gauge-dependent condensates $\left\langle\Phi_{a}\right\rangle$, which is formally at odds with Elitzur's theorem [38]. These should be read as stand-in for the theory being in a Higgs phase.

[38] S. Elitzur, Phys. Rev. D 12, 3978 (1975).

[39] D. Finnell and P. Pouliot, Nucl. Phys. B453, 225 (1995).

[40] The overall number of Nambu-Goldstone (NG) superfields is given by $\frac{1}{2} \operatorname{dim}\left(G^{c} / \tilde{H}\right)$ [41], where $G^{c}$ is the complexification of the original global symmetry $G$, and $\tilde{H} \supseteq H^{c}$ depends on the representation of the VEV. When $\tilde{H}=H^{c}$, each NG superfield contains one NGB. When $\tilde{H} \supset H^{c}$, there are fewer NG superfields, and hence some of them contain two NGBs to account for all the broken generators 
of $G / H$. In our case, we can do the counting in two steps. First we consider $S U(N-4) / S U(N-5) \times U(1)$ which gives the Kähler manifold $\mathbb{C} P^{N-5}$ and $N-5$ chiral superfields, each containing two NGBs. In the second step we consider $S U(N-5) \times U(1) / S p(N-5)$, which gives $\frac{1}{2}(N-5)(N-6)$ chiral superfields, each with a single NGB and its superpartner. Overall, we get $\frac{1}{2}(N-5)(N-4)$, which is exactly the number of uneaten, $S U(5)$ neutral chiral superfields.

[41] W. Lerche, Nucl. Phys. B238, 582 (1984).
[42] Note that this notation is not a chiral superfield, but rather an interpolating field for the massless fermions in $\bar{F}_{N-4}$ written as gauge-invariant operators. This is consistent as supersymmetry is dynamically broken.

[43] J. Wess and B. Zumino, Phys. Lett. 37B, 95 (1971).

[44] E. Witten, Nucl. Phys. B223, 422 (1983).

[45] E. D'Hoker and E. Farhi, Nucl. Phys. B248, 59 (1984).

[46] I. Affleck, M. Dine, and N. Seiberg, Nucl. Phys. B256, 557 (1985). 